Article

\title{
ESR Estimation Schemes of Output Capacitor for Buck Converter from Capacitor Perspective
}

\author{
Lei Ren ${ }^{1, *} \mathbb{0}$, Lei Zhang $1, * \mathbb{C}$ and Chunying Gong ${ }^{2, *}$ \\ College of Electrical Engineering, Nantong University, Nantong 226019, China \\ 2 College of Automation, Nanjing University of Aeronautics and Astronautics, Nanjing 210016, China \\ * Correspondence: ee_renlei@ntu.edu.cn (L.R.); nttzzl@ntu.edu.cn (L.Z.); zjnjgcy@nuaa.edu.cn (C.G.)
}

Received: 24 August 2020; Accepted: 28 September 2020; Published: 29 September 2020

\begin{abstract}
The aluminum electrolytic capacitor (AEC) is one of the most vulnerable parts in power electronic converters and its reliability is crucial to the whole system. With the growth of service time, the equivalent series resistance (ESR) increases and the capacitance (C) decreases due to the loss of electrolytes, which will result in extra power loss and even damage to transistors. To prevent significant damages, the AEC must be replaced at an optimal period and online health monitoring is indispensable. Through the analysis of degradation parameters (ESR and C), ESR is proved to be a better health indicator and therefore is determined as the monitoring parameter for AEC. From the capacitor perspective, ESR estimation schemes of output capacitors for a Buck converter are studied. Based on the voltage-current characteristics, two ESR calculation models are proposed, which are applicable for both continuous conduction mode (CCM) and discontinuous conduction mode (DCM). From the point of implementation view, the advantages and disadvantages of the two estimation schemes are pointed out, respectively. A Buck prototype is built and tested, and simulation and experimental results are provided to validate the proposed ESR estimation schemes.
\end{abstract}

Keywords: aluminum electrolytic capacitor (AEC); health monitoring; ESR estimation; Buck converter

\section{Introduction}

For the advantages of high power, density and efficiency, power electronic converters (PECs) have been widely used in many different fields such as new energy, industry and aviation [1-4]. However, PECs have synthetic complexities such as high stress and strong nonlinearity, which causes challenges to their operational reliability. To suppress high-frequency voltage ripples and keep output voltage steady, power filter capacitors are indispensable for PECs. Owing to the high capacitance per volume and low cost, aluminum electrolytic capacitors (AECs) are usually the preferred capacitors. Unfortunately, it is reported that AECs are the most vulnerable parts in PECs [5,6]. The evaporation of the electrolyte is the main degradation pattern of AECs, which will increase the equivalent series resistance (ESR) and decrease the capacitance (C) gradually. These degradations will increase voltage and current ripples, producing more power loss and even device damage. Moreover, prolonged use of aged capacitors will lead to system failure once the electrolyte is dried up, increasing the cost of maintenance and affecting the normal production. To prevent significant damages, AECs must be monitored in real time and replaced at an optimal period. According to the manufactures, C and ESR can be used to indicate the health status of AECs. Generally, the service life of an AEC is considered to be over once the $C$ decreases to $80 \%$ or the ESR increases to more than twice the initial value under the same condition $[7,8]$. Therefore, online ESR or C estimation is critical for condition monitoring of AECs.

In the literature, a lot of online schemes have been proposed to calculate $\mathrm{C}$ and/or ESR for condition monitoring of AECs. From the perspective of the circuit, the existing methods can be summarized as 
system perspective methods (SPMs) and capacitor perspective methods (CPMs). In SPMs, multiple components besides AECs are monitored at the same time and parameter identification is often used. Circuit model is the basis of parameter identification, which determines the accuracy of identification results. In [9-12], the state-space averaging model is used and the parameter identification is conducted by the means of signal injection. However, these methods have the shortcomings of high computational complexity, which restricts its real application. To avoid signal injection, a hybrid model is proposed for parameter identification by measuring the switch states of a converter in $[13,14]$. However, the driving and switching delay time of the actual device increase the difficulty of switch states measurement and will cause identification error.

Compared to SPMs, CPMs can achieve higher identification precision by fewer sensors. In CPMs, the ripple voltage and/or current of AEC are needed for ESR and C calculations. Since most of the switching ripple current flows through the capacitor, this ripple current is directly used to estimate ESR in [15-17]. In [15], capacitor voltage and current ripples are extracted by a band-pass filter and ESR is calculated at the centre frequency. In [16], the inductor current derivative is obtained by a Rogowski coil-based sensor for ESR calculation. In [17], Empirical Mode Decomposition (EMD) is used to extract ripples for ESR estimation. However, these methods are relatively complex in hardware or software implementation. To reduce the use of sensors, current-sensorless monitoring methods of $C$ and ESR are proposed for a continuous conduction mode (CCM) Buck converter, for a CCM flyback converter and a discontinuous conduction mode (DCM) flyback converter, respectively in [18-20]. Similar methods are applied to a CCM boost converter in [21] and realized for DCM in [22]. However, these current-sensorless methods are affected by other parameters such as the duty cycle and filter inductance. If the duty cycle is measured inaccurately or the filter inductance is changed by aging, estimation error will be produced. Beside these direct methods, power loss is another way to estimate ESR [23]. Power dissipation is calculated by measuring RMS values of the capacitor current and the capacitor voltage. However, it is reported that the capacitor voltage is distorted by the capacitor current sensor in [24]. Moreover, the low-pass filter introduced in the power loss calculation will cause error [23]. In [25,26], the load current measurement is added for C estimation through the transient analysis of output voltage.

To address the aforementioned issues, ESR is chosen as the monitoring parameter for AEC and two ESR calculation models are derived for Buck converters from a capacitor perspective in this paper. The proposed models apply to both CCM and DCM. This paper is organized as follows. In Section 2, the degradation parameters (ESR and C) of AECs are analyzed and ESR is determined as the monitoring parameter for AEC in this paper. In Section 3, ESR estimation schemes are studied from a capacitor perspective and two ESR estimation models are proposed. Simulation studies are carried out for model validation on MATLAB in Section 4. Experimental results are presented in Sections 5 and 6 concludes the paper.

\section{Analysis of Degradation Parameters}

In general, $\mathrm{C}$ and ESR are used as the indicators of degradation AECs. However, the values of ESR and $C$ are affected not only by aging but also temperature and frequency. Figure $1 \mathrm{a}, \mathrm{b}$ show the frequency characteristic curves of C and ESR for capacitor CD297 450V680 $\mu$ F (Jianghai, Nantong, China) at different temperatures. The frequency characteristic curves of $C$ and ESR are affected by the dielectric absorption and dielectric dissipation factors of AEC, which are mainly determined by the frequency characteristics of aluminum oxide film and electrolyte. From Figure $1 \mathrm{a}$, it can be seen that $\mathrm{C}$ presents two different characteristics in high temperature zone $\left(45 \sim 85^{\circ} \mathrm{C}\right)$ and low temperature zone $\left(-40 \sim 25^{\circ} \mathrm{C}\right)$. In the high temperature zone, the capacitance increases as the frequency increases, and in the low temperature zone, the capacitance decreases as the frequency increases. Moreover, at the same frequency, the capacitance presents a non-monotonic temperature characteristic. From Figure 1b, it can be seen that the ESR $-f$ curve has good temperature and frequency monotonicity: ESR decreases as frequency increases and decreases as temperature increases. If a converter operates at a temperature of 
$0 \sim 85^{\circ} \mathrm{C}$ and at a frequency of $10 \sim 100 \mathrm{kHz}$, it can be found that ESR has lower temperature sensibility and lower frequency sensibility than C. Not only that, ESR also has higher degradation sensibility, which has been indicated in $[7,8]$. The accelerated aging test results at $120 \mathrm{~Hz}$ are presented in Figure 2, where it can be found that the relative variations of ESR are much larger than that of $C$. As a matter of fact, the evaporation of the electrolyte is the main degradation pattern of AEC. The evaporation of the electrolyte will cause an increase in ESR directly and $C$ is mainly affected by the thickness of alumina film. In the service life of an AEC, the variation of $C$ is not permitted beyond $20 \%$ but the variation of ESR usually exceeds $100 \%$. Therefore, ESR is chosen as the optimum degradation parameter for AECs.

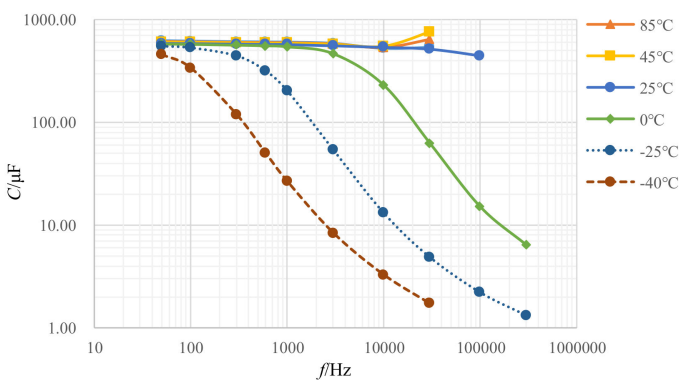

(a)

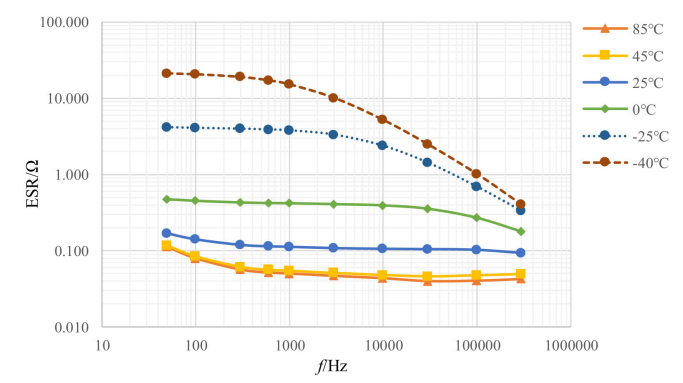

(b)

Figure 1. The frequency characteristic curves of capacitance (C) and equivalent series resistance (ESR) for capacitor CD297 450V680 $\mathrm{FF}$ : (a) C-f; (b) ESR-f.

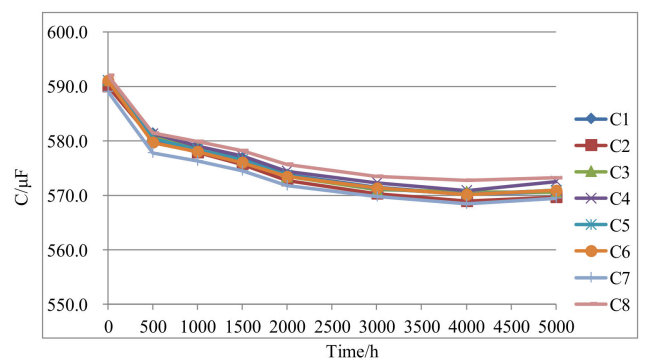

(a)

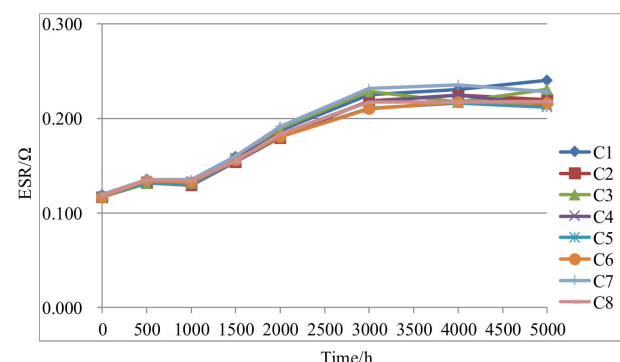

(b)

Figure 2. The accelerated aging test results of CD297 450V680 $\mu \mathrm{F}$ : (a) capacitance data; (b) ESR data.

\section{ESR Estimation Schemes}

In power electronics converters, voltage ripples and current ripples are the basis for C and ESR calculation. Therefore, it is necessary to analyze the ripple characteristic. In general, from capacitor perspective, the output structure of power electronic converter can be illustrated as shown in Figure 3, where $i_{\mathrm{ls}}$ is the total current of the capacitor branch and load branch, $i_{\mathrm{C}}$ is the current of the capacitor branch, the electrolytic capacitor $C_{\mathrm{f}}$ is equivalent to a capacitance $C$ in series with a resistance ESR, and $R_{\mathrm{O}}$ is the output load.

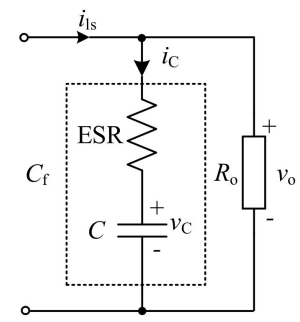

Figure 3. Output structure of power electronic converters. 
In such a structure, the output voltage ripple $v_{\text {o_ac }}$ can be divided into two components

$$
v_{\mathrm{O} \_\mathrm{ac}}=v_{\mathrm{ESR}}+v_{\mathrm{C} \_\mathrm{ac}}
$$

where $v_{\mathrm{ESR}}$ and $v_{\mathrm{C} \_ \text {ac }}$ are the ripple voltages across $\mathrm{ESR}$ and $\mathrm{C}$, respectively.

According to the characteristics of resistance and capacitance, the two components have a relationship as

$$
C \frac{\mathrm{d} v_{\mathrm{C}_{-} \mathrm{ac}}}{\mathrm{d} t}=\frac{v_{\mathrm{ESR}}}{\mathrm{ESR}}=i_{\mathrm{C}}
$$

If $v_{\mathrm{ESR}}$ and $i_{\mathrm{C}}$ are extracted, ESR can be calculated directly. However, in actual circuit, only $v_{\mathrm{O}_{-} \text {ac }}\left(\right.$ a total of $v_{\mathrm{ESR}}$ and $v_{\mathrm{C}_{-} \text {ac }}$ ) and $i_{\mathrm{C}}$ can be acquired. Therefore, the key of ESR estimation is the separation of $v_{\mathrm{ESR}}$ from $v_{\mathrm{O} \_a c}$, which can be regarded as a signal processing issue. The existing methods of signal processing can be classified into frequency-domain approaches and time-domain approaches. The frequency-domain approaches can only separate different frequency components, which are difficult to extract $v_{\mathrm{ESR}}$ from $v_{\mathrm{O} \_a c}$ at the same frequency. For this reason, time-domain analysis is carried out in the following.

In general, the impedance of the electrolytic capacitor at the switching frequency is much less than that of the load. In this situation, almost all the switching frequency ripple current $i_{\text {ls_ac }}$ flows through the capacitor, which can be described as

$$
i_{\mathrm{C}} \approx i_{\mathrm{ls} \_\mathrm{ac}}
$$

Notably, this formula is the presupposition of the proposed calculation models in this paper, which indicates that the following proposed models are applicable as long as the impedance of the load is much larger than the impedance of AEC at the switching frequency. Additionally, for the sake of analysis, resistive load is adopted for the following model derivation.

\subsection{ESR Estimation Schemes Analysis}

The representative waveforms of the Buck converter in CCM and DCM are shown in Figure 4a,b, respectively, where $v_{\mathrm{gs}}$ is the driving signal, $v_{\mathrm{ESR}}$ represents the voltage across the ESR (the capacitor current $\left.i_{C}\right), v_{C}$ represents the voltage across the pure capacitance, and $v_{\mathrm{o}}$ is the output voltage. From Figure 4, two characteristics can be observed: 1. there are two different moments that $v_{C}$ has the same amplitude; 2 . $v_{\mathrm{C}_{-} \text {ac }}$ is orthogonal to $v_{\mathrm{ESR}}$ on the interval $[0, T]$.

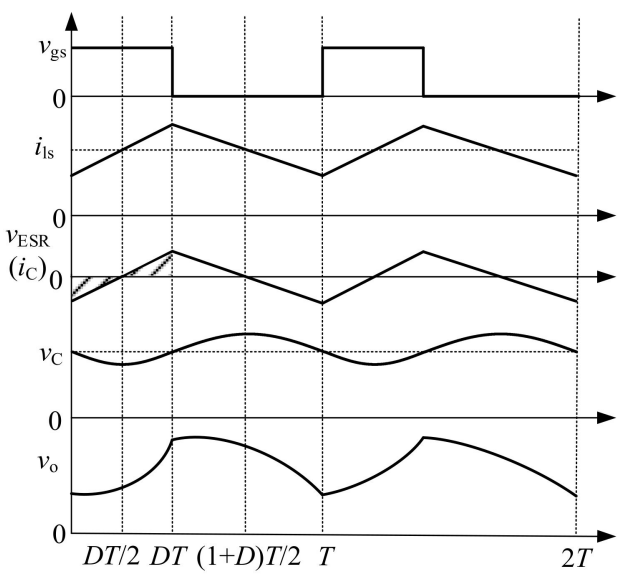

(a)

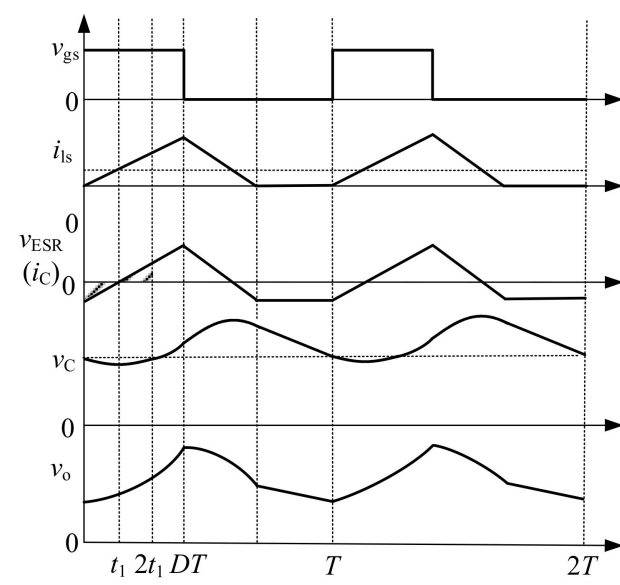

(b)

Figure 4. Representative waveforms of Buck converter: (a) continuous conduction mode (CCM); (b) discontinuous conduction mode (DCM). 
3.1.1. Scheme 1: Based on the Specific Moments

According to (1) and (2), ESR can be calculated by

$$
\begin{gathered}
\Delta v_{\mathrm{o}_{\_} \mathrm{ac}}=\Delta v_{\mathrm{ESR}}+\Delta v_{\mathrm{C} \_\mathrm{ac}}, \\
\mathrm{ESR}=\frac{\Delta v_{\mathrm{ESR}}}{\Delta i_{\mathrm{C}}},
\end{gathered}
$$

From (4) and (5), it can be seen that ESR can be calculated by $\Delta v_{\mathrm{o}_{-} a d} / \Delta i_{\mathrm{C}}$ when $\Delta v_{\mathrm{C}_{-} \mathrm{ac}}=0$. Therefore, ESR can be obtained easily if the extracted two moments satisfy $\Delta v_{\mathrm{C}_{-} \text {ac }}=0$. In CCM, $i_{\mathrm{C}}$ is a triangular wave and $i_{C}(D T / 2)$ is at the midpoint of the rising edge, namely $v_{C}(0)=v_{C}(D T)$. In DCM, $t_{1}$ is the zero crossing point of $i_{C}$ and $2 t_{1}$ is less than $D T$ obviously. As a result, there is also a moment $2 t_{1}$ that satisfies $v_{C}(0)=v_{C}\left(2 t_{1}\right)$ in DCM.

In conclusion, ESR calculation model can be derived as

$$
\mathrm{ESR}=\frac{v_{\mathrm{O}_{-} \mathrm{ac}}(0)-v_{\mathrm{O} \_\mathrm{ac}}(x)}{i_{\mathrm{C}}(0)-i_{\mathrm{C}}(x)},
$$

where $x=D T$ in CCM and $x=2 t_{1}$ in DCM.

\subsubsection{Scheme 2: Based on the Orthogonality}

For the reason that it only contains ac components and satisfies the Dirichlet principle, $i_{C}(t)$ can be expressed in the form of Fourier as

$$
i_{C}(t)=\sum_{n=1}^{\infty}\left(a_{n} \cos \left(2 n \pi f_{\mathrm{s}} t\right)+b_{\mathrm{n}} \sin \left(2 n \pi f_{\mathrm{s}} t\right)\right),
$$

where $f_{\mathrm{s}}$ represents the switching frequency. Substituting $(7)$ into $(2), v_{\mathrm{C}_{-} \text {ac }}$ can be obtained as

$$
v_{\mathrm{C}_{-} \mathrm{ac}}(t)=\frac{1}{2 \pi f_{\mathrm{s}} \mathrm{C}} \sum_{n=1}^{\infty} \frac{1}{n}\left(a_{\mathrm{n}} \sin \left(2 n \pi f_{\mathrm{s}} t\right)-b_{\mathrm{n}} \cos \left(2 n \pi f_{\mathrm{s}} t\right)\right),
$$

It can be found that

$$
\int_{0}^{T} i_{\mathrm{C}}(t) v_{\mathrm{C} \_\mathrm{ac}}(t) d t=\frac{1}{2 \pi f_{\mathrm{s}} C} \sum_{n=1}^{\infty} \int_{0}^{\frac{1}{f_{\mathrm{s}}}} \frac{1}{n}\left[\begin{array}{c}
\frac{a_{\mathrm{n}}^{2}-b_{\mathrm{n}}^{2}}{2} \sin \left(4 n \pi f_{\mathrm{s}} t\right) \\
-a_{\mathrm{n}} b_{\mathrm{n}} \cos \left(4 n \pi f_{\mathrm{s}} t\right)
\end{array}\right]=0,
$$

which proves the orthogonality of $v_{C_{-} \text {ac }}$ and $v_{\mathrm{ESR}}\left(i_{\mathrm{C}}\right)$ on the interval $[0, T]$. Moreover, similarly, if the equivalent series inductance (ESL) is considered, the ac voltage across ESL is also orthogonal to $v_{\mathrm{ESR}}\left(i_{\mathrm{C}}\right)$ on the interval $[0, T]$, which means that the ESL of AEC does not affect the results.

Moreover, it can be further derived that

$$
\int_{0}^{T} i_{\mathrm{C}}(t) v_{\mathrm{O} \_\mathrm{ac}}(t) d t=\int_{0}^{T} i_{\mathrm{C}}(t)\left(v_{\mathrm{C}}(t)+v_{\mathrm{ESR}}(t)\right) d t=\int_{0}^{T} i_{\mathrm{C}}(t)\left(v_{\mathrm{C}}(t)+\operatorname{ESR}_{\mathrm{C}}(t)\right) d t=\operatorname{ESR} \int_{0}^{T} i_{\mathrm{C}}^{2}(t) d t,
$$

Thus, the calculation model of ESR can be presented finally as

$$
\operatorname{ESR}=\frac{\int_{0}^{T} i_{C}(t) v_{\mathrm{o} \_a c}(t) d t}{\int_{0}^{T} i_{\mathrm{C}}^{2}(t) d t}
$$

The numerator of (11) is the ac loss of AEC in one switching cycle. In a power electronic converter, $i_{C}$ is almost unaffected by ESR variation, which means the ac loss of AEC will increase while ESR increases and it is important to monitor the ESR variation. 


\subsection{ESR Estimation Implementations}

Two ESR estimation schemes are revealed by (6) and (11), respectively, and there are various ways to realize them. In this part, we will provide a simple and efficient solution for each scheme.

\subsubsection{Implementation of Scheme 1}

In scheme $1, t_{1}$ in DCM is difficult to obtain and $i_{1 \mathrm{ls} \_ \text {ac }}$ needs to be measured to capture $t_{1}$. To reduce the number of measured signals, $i_{C}$ in (6) can be replaced by $i_{1 \mathrm{~s} \_a c}$ as

$$
\mathrm{ESR}=\frac{v_{\mathrm{O} \_\mathrm{ac}}(0)-v_{\mathrm{O} \_\mathrm{ac}}(x)}{i_{\mathrm{ls} \_\mathrm{ac}}(0)-i_{\mathrm{ls} \_\mathrm{ac}}(x)},
$$

Actually, these specific moments can be obtained by capturing the zero crossing moments of $v_{C_{-}} a c$ and (12) can be transformed into

$$
\mathrm{ESR}=\frac{v_{\mathrm{o} \_a c}\left(t_{z 1}\right)-v_{\mathrm{O} \_a c}\left(t_{z 2}\right)}{i_{\mathrm{ls} \_a c}\left(t_{z 1}\right)-i_{\mathrm{ls} \_a c}\left(t_{z 2}\right)}
$$

where $t_{\mathrm{z} 1}$ and $t_{\mathrm{z} 2}$ are the two continuous zero crossing points of $v_{\mathrm{C}_{-} \mathrm{ac}} \cdot t_{\mathrm{z} 1}$ and $t_{\mathrm{z} 2}$ can be captured by extracting the zero crossing points of the integral for $i_{\mathrm{ls} \_a c}$. However, the integral often introduces $\mathrm{dc}$ components, which will lead to the extraction errors. To avoid this defect, Hilbert transform is used to replace the integral. Hilbert transform is a signal processing method that can achieve a 90-degree move-phase at all frequencies. Hilbert transform is realized by using the "hilbert" function on MATLAB, which is used to obtain the analytic signal of $i_{1 \mathrm{~s} \_a c}$ :

$$
\operatorname{hilbert}\left(i_{\mathrm{ls} \_a c}\right)=v_{\mathrm{o} \_a c}+i \times \operatorname{Hilbert}\left(i_{\mathrm{ls} \_a c}\right)
$$

where Hilbert $\left(i_{\mathrm{ls} \_a c}\right)$ is the actual Hilbert transform of $i_{\mathrm{ls} \_a c}$ and is acquired by the "imag" function.

\subsubsection{Implementation of Scheme 2}

To implement scheme 2, the ESR calculation model of (11) can be discretized into

$$
\mathrm{ESR}=\frac{\sum_{i=1}^{N} I_{1 \mathrm{~s} \_\mathrm{ac}}(i) V_{\mathrm{o} \_a c}(i)}{\sum_{i=1}^{N} I_{\mathrm{ls} \_\mathrm{ac}}^{2}(i)},
$$

where $N$ is sampling number and $N$ should cover several switching periods.

\section{Simulation Study}

For verifying effectiveness of the proposed ESR calculation models, the simulation model of Buck converter is built on MATLAB/SIMULINK, and the circuit parameters are listed in Table 1. $R_{\mathrm{o}}=10 \Omega$ and $R_{\mathrm{O}}=100 \Omega$ are used for CCM simulation and DCM simulation, respectively.

Table 1. Simulation Parameters of Buck Converter.

\begin{tabular}{ccc}
\hline Parameters & Variable & Value \\
\hline Input voltage & $V_{\mathrm{in}} / \mathrm{V}$ & 24 \\
Inductance & $L_{\mathrm{f}} / \mu \mathrm{H}$ & 220 \\
Output capacitor & $\mathrm{C}_{\mathrm{f}} / \mu \mathrm{F}$ & 100 \\
Output load resistor & $R_{\mathrm{o}} / \Omega$ & $10(\mathrm{CCM}), 100(\mathrm{DCM})$ \\
ESR of output capacitor & $\mathrm{ESR} / \Omega$ & 0.2 \\
Duty cycle & $D$ & 0.5 \\
Switching frequency & $f_{\mathrm{s}} / \mathrm{kHz}$ & 50 \\
Sampling frequency & $f_{\mathrm{sa}} / \mathrm{MHz}$ & 10 \\
\hline
\end{tabular}


For the proposed two calculation models, the sampled signals are both the inductor ac current $i_{\mathrm{Lf} \_a c}$ and the output ac voltage $v_{\mathrm{O} \_a c}$. The waveforms of $i_{\mathrm{Lf} \_a c}$ and $v_{\mathrm{O} \_a c}$ in CCM and DCM are shown in Figure 5, respectively.

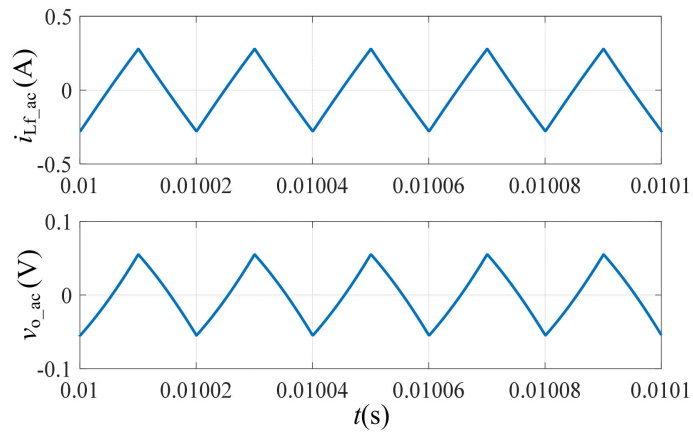

(a)

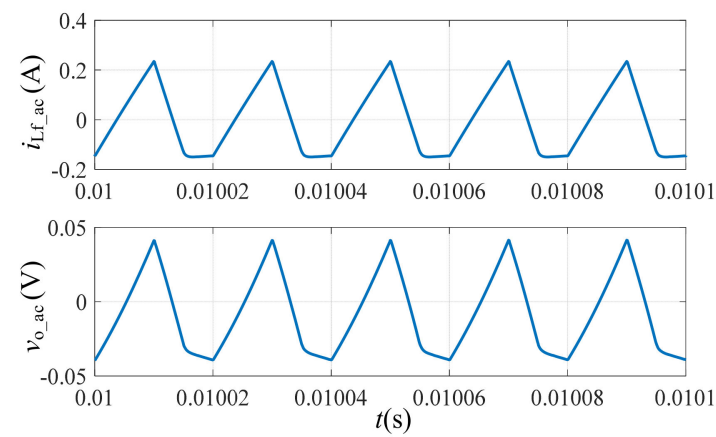

(b)

Figure 5. Simulation waveforms of Buck converter in ideal condition: (a) CCM; (b) DCM.

\subsection{Method 1}

Figure 6 shows the signal processing waveforms and Table 2 lists the ESR estimation results calculated by (13) in CCM and DCM. The estimation error is about $1 \%$ and the estimation error in DCM is a little smaller than that in CCM.
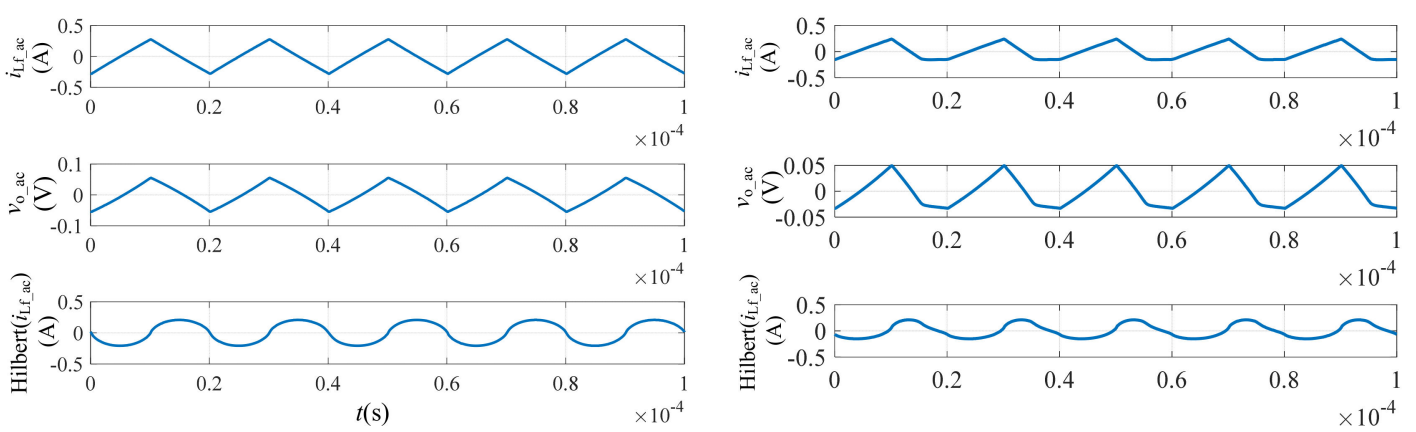

(a)

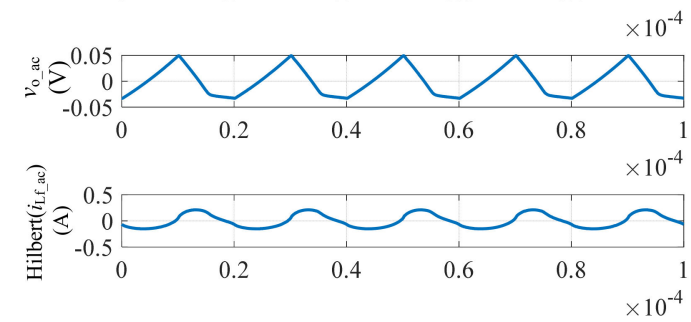

(b)

Figure 6. Signal processing waveforms of Buck converter in ideal condition: (a) CCM; (b) DCM.

Table 2. ESR Simulation Results of Buck Converter by Method 1.

\begin{tabular}{ccccc}
\hline Reference & \multicolumn{4}{c}{ Simulation Results } \\
\hline \multirow{2}{*}{ ESR $/ \Omega$} & $\mathrm{CCM}\left(\boldsymbol{R}_{\mathbf{o}}=\mathbf{1 0} \Omega\right)$ & $\mathrm{DCM}\left(\boldsymbol{R}_{\mathbf{0}}=\mathbf{1 0 0} \Omega\right)$ \\
\cline { 2 - 5 } & $\mathrm{ESR} / \Omega$ & error $/ \%$ & $\mathrm{ESR} / \Omega$ & error $/ \%$ \\
\hline 0.2 & 0.1975 & 1.25 & 0.2020 & 1 \\
\hline
\end{tabular}

The advantages of method 1 is that it only needs to capture two points for a calculation and it can reserve low computational complexity. However, it is precisely because the data used for calculation are few that this method is sensitive to noise, which means it requires high-accuracy sampling and a good de-noising method.

\subsection{Method 2}

Table 3 lists the ESR estimation results calculated by (15). For each calculation, 1000 data points (five switching cycles) are acquired and ESR is calculated by 200, 400, 600, 800 and 1000 points, respectively, from top to bottom. It can be seen that the simulation results are a little smaller than 
the reference, whether in CCM or DCM. This is caused by the approximation of (3). In fact, some of $i_{\text {ls_ac }}$ flows through the load resistance and the actual calculation value is ESR// $R_{\mathrm{o}}$. For the same ESR, the larger $R_{\mathrm{O}}$ is, the smaller error is. In given $R_{\mathrm{O}}$ circumstance, the calculated results can be corrected correspondingly.

Table 3. ESR Simulation Results of Buck Converter by Method 2.

\begin{tabular}{ccccc}
\hline Reference & \multicolumn{4}{c}{ Simulation Results } \\
\hline \multirow{2}{*}{ ESR $/ \boldsymbol{\Omega}$} & $\mathbf{C C M}\left(\boldsymbol{R}_{\mathbf{o}}=\mathbf{1 0} \boldsymbol{\Omega}\right)$ & DCM $\left(\boldsymbol{R}_{\mathbf{o}}=\mathbf{1 0 0} \boldsymbol{\Omega}\right)$ \\
\cline { 2 - 5 } & $\mathrm{ESR} / \boldsymbol{\Omega}$ & error/\% & ESR $/ \boldsymbol{\Omega}$ & error $/ \%$ \\
\hline \multirow{2}{*}{0.2} & 0.1962 & 1.9 & 0.1999 & 0.05 \\
& 0.1962 & 1.9 & 0.1997 & 0.15 \\
& 0.1962 & 1.9 & 0.1996 & 0.20 \\
& 0.1962 & 1.9 & 0.1996 & 0.20 \\
& 0.1962 & 1.9 & 0.1996 & 0.20 \\
\hline
\end{tabular}

Compared to method 1, method 2 requires more data for calculation and needs more computation. However, method 2 can get a better anti-noise property for the use of more sampling data and can reduce the requirement for the sampling device.

\section{Experimental Results}

To validate the proposed schemes, a Buck converter is assembled in the laboratory. The Buck converter is open-loop controlled and the output voltage is regulated by the duty cycle of MOSFET, which is realized by IC SG3525 (Motorala, Tokyo, Japan). The driving signal of MOSFET is produced by IC IXDN609PI (IXYS, Milpitas, CA, USA). The main specifications are as follows: input voltage: $24 \mathrm{~V}$; output voltage: $12 \mathrm{~V}$; switching frequency: $44.5 \mathrm{kHz}$; power diode: IXYS DSEP60-06A (IXYS, Milpitas, CA, USA); power MOSFET: IXYS IXFK64N50P (IXYS, Milpitas, CA, USA); output AEC: CAPXON 100V100 $\mu$ F (CapXon, Shenzhen, China); load resistance $R_{\mathrm{o}}: 10 \Omega / 400 \Omega$. The waveform data are acquired by Agilent DSO-X 2014a (Agilent Technologies, Santa Clara, CA, USA) and the sampling frequency is $100 \mathrm{MHz}$. The collected data are uploaded to the computer and ESR is calculated on MATLAB 2016b (MathWorks, Natick, MA, USA). The reference values are measured offline by LCR meter at the switching frequency before the capacitor is assembled to the converter at $25^{\circ} \mathrm{C}$.

The sampled experimental waveforms are shown in Figure 7. It can be seen that the collected signals are mixed with much noise, especially $v_{\mathrm{o}_{\mathrm{a}} \mathrm{ac}}$, which will cause large estimation errors and even estimation failure. To eliminate the influence of noise on estimation results, a wavelet threshold de-noising method is adopted. The effect of the wavelet de-noising method mainly depends on the choice of wavelet generating function, the level of decomposition, and the setting of threshold. Synthesizing the maximum signal-noise ratio principle and calculation efficiency, Wavelet Coiflets 5 is chosen, the level of decomposition is set as 3 and fixed threshold mode is adopted. The de-noised waveforms are shown in Figure 8 and these de-noised waveforms are used for ESR estimation.

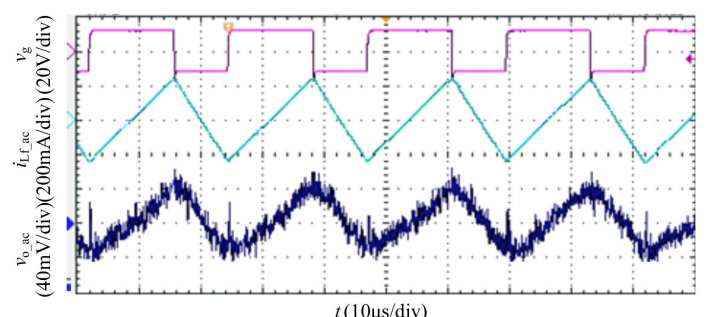

(a)

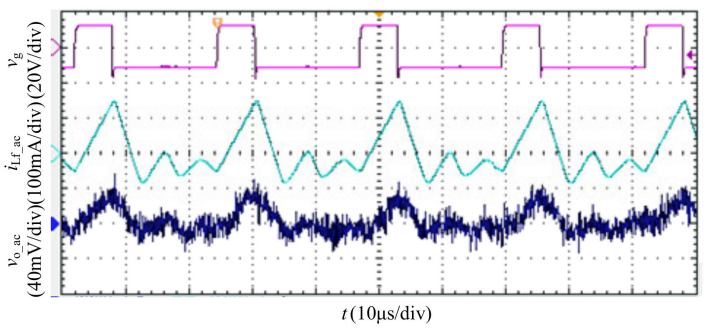

(b)

Figure 7. Experimental waveforms of Buck converter before de-noising: (a) CCM; (b) DCM. 

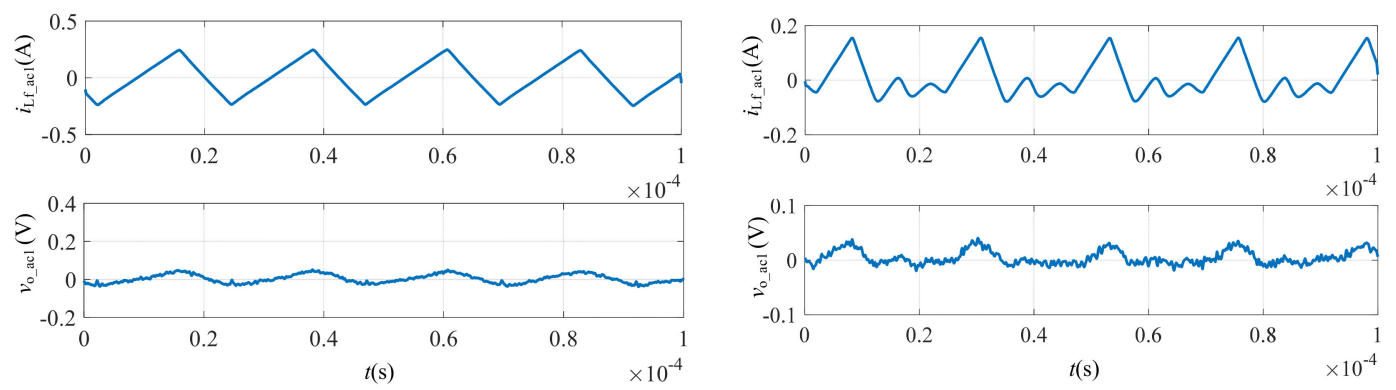

(a)

(b)

Figure 8. Experimental waveforms of Buck converter after de-noising: (a) CCM; (b) DCM.

\subsection{Method 1}

To calculate ESR by method 1, the Hilbert transform of the inductor ac current is carried out and the waveforms of CCM and DCM are shown in Figure 9, respectively. The dotted boxes in the figure depict the zero-crossing points and the actual calculation times (5 times in CCM and 9 times in DCM). The experimental ESR estimation results of method 1 are listed in Table 4.
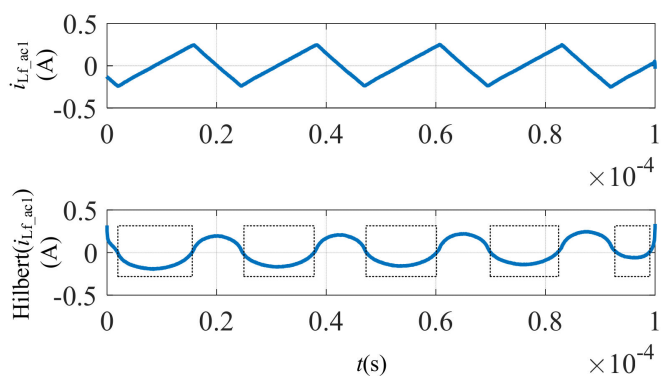

(a)
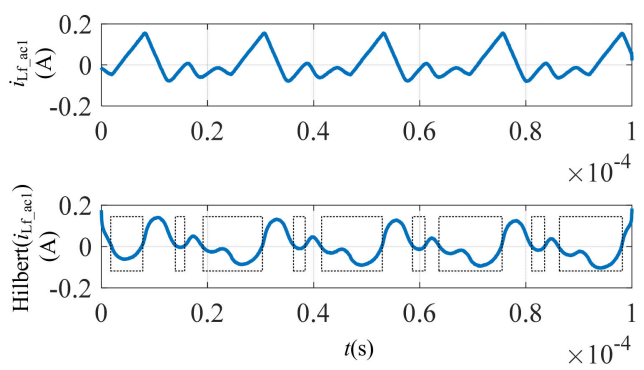

(b)

Figure 9. The de-noised ac component of the inductor current and its Hilbert transform: (a) CCM; (b) DCM.

Table 4. ESR Experimental Results of Buck Converter by Method 1 after De-noising.

\begin{tabular}{|c|c|c|c|c|}
\hline \multirow{3}{*}{$\begin{array}{c}\text { Reference } \\
\mathrm{ESR} / \Omega\end{array}$} & \multicolumn{4}{|c|}{ Experimental Results } \\
\hline & \multicolumn{2}{|c|}{$\operatorname{CCM}\left(R_{\mathrm{o}}=10 \Omega\right)$} & \multicolumn{2}{|c|}{$\operatorname{DCM}\left(R_{\mathrm{o}}=400 \Omega\right)$} \\
\hline & $\mathrm{ESR} / \Omega$ & error/\% & $\mathrm{ESR} / \Omega$ & error/\% \\
\hline \multirow{9}{*}{0.1922} & 0.1733 & 9.8 & 0.1846 & 3.9 \\
\hline & 0.1815 & 5.6 & 0.2133 & 10.9 \\
\hline & 0.1823 & 5.2 & 0.1818 & 5.4 \\
\hline & 0.1798 & 6.5 & 0.2147 & 11.7 \\
\hline & 0.1855 & 3.5 & 0.1799 & 6.4 \\
\hline & & \multirow{4}{*}{ - } & 0.2153 & 12.0 \\
\hline & & & 0.1777 & 7.5 \\
\hline & & & 0.2155 & 12.1 \\
\hline & & & 0.1789 & 6.9 \\
\hline
\end{tabular}

As the results are calculated by the data of two specific moments, the estimation results are easily affected by the noise and fluctuate obviously. From Figure 8, it can found that the signal-noise ratio of $v_{\mathrm{O} \_a c 1}$ in DCM is smaller than that in CCM, which agrees well with the higher volatility in the estimation results in DCM. By comparing the results of narrow and wide time intervals in DCM, it shows that 
the results in wide time intervals have higher precision and this is because the signals in wide time intervals have higher signal-noise ratio. Overall, this scheme has bad identification stability in low signal-to-noise ratio conditions and it is suitable for high-precision sampling and low-complexity computation occasions.

\subsection{Method 2}

The experimental results of method 2 are listed in Table 5. ESR is calculated with one cycle $(N=2247)$ to eight cycles $(N=8988)$. In comparison with the results of CCM, the results in DCM are closer to the reference. This is caused by the approximation of (3), which agrees well with the simulation. Moreover, the cross correlation coefficient of the noise is usually very small, which means this method has good noise immunity. To verify the noise immunity, ESR estimation results calculated by using the signals before de-noising are listed in Table 6. Compared to the results in Table 5, the difference of the results in Table 6 is small, which verifies the good noise immunity of this method. Overall, this scheme has good identification precision and stability.

Table 5. ESR Experimental Results of Buck Converter by Method 2 after De-noising.

\begin{tabular}{|c|c|c|c|c|}
\hline \multirow{3}{*}{$\begin{array}{c}\text { Reference } \\
\mathrm{ESR} / \Omega\end{array}$} & \multicolumn{4}{|c|}{ Experimental Results } \\
\hline & \multicolumn{2}{|c|}{$\operatorname{CCM}\left(R_{0}=10 \Omega\right)$} & \multicolumn{2}{|c|}{$\operatorname{DCM}\left(R_{\mathrm{o}}=400 \Omega\right)$} \\
\hline & $\mathrm{ESR} / \Omega$ & error/\% & $\mathrm{ESR} / \Omega$ & error/\% \\
\hline \multirow{4}{*}{0.1922} & 0.1819 & 5.3 & 0.1994 & 3.7 \\
\hline & 0.1813 & 5.7 & 0.2004 & 4.3 \\
\hline & 0.1763 & 8.3 & 0.1882 & 2.1 \\
\hline & 0.1767 & 8.1 & 0.1878 & 2.3 \\
\hline
\end{tabular}

Table 6. ESR Experimental Results of Buck Converter by Method 2 before De-noising.

\begin{tabular}{|c|c|c|c|c|}
\hline \multirow{3}{*}{$\begin{array}{c}\text { Reference } \\
\text { ESR } / \Omega\end{array}$} & \multicolumn{4}{|c|}{ Experimental Results } \\
\hline & \multicolumn{2}{|c|}{$\operatorname{CCM}\left(R_{0}=10 \Omega\right)$} & \multicolumn{2}{|c|}{$\operatorname{DCM}\left(R_{\mathrm{o}}=400 \Omega\right)$} \\
\hline & $\mathrm{ESR} / \Omega$ & error/\% & $\mathrm{ESR} / \Omega$ & error/\% \\
\hline \multirow{4}{*}{0.1922} & 0.1810 & 5.8 & 0.1917 & 0.3 \\
\hline & 0.1802 & 6.2 & 0.2002 & 4.2 \\
\hline & 0.1751 & 8.9 & 0.1879 & 2.2 \\
\hline & 0.1755 & 8.7 & 0.1875 & 2.4 \\
\hline
\end{tabular}

\subsection{Error Analysis}

In addition to the load resistance, the estimation error of the proposed methods is also induced by other factors:

1. Noise effect. Though the signal-to-noise ratio is greatly improved by the wavelet de-noising method, noise cannot be completely eliminated and the effective signal is also attenuated.

2. Sampling resolution. The estimation precision is determined by the sampling resolution of voltage and current ripples. However, the voltage and current ripples are small, which needs equipment with high resolution.

3. Errors of the reference values. Errors also exist in the measured reference values and only values at a certain frequency can be measured by LCR meter. However, the used inductor current and capacitor voltage for ESR estimation not only contain switching-frequency components, but also an amount of harmonic components, which will lead to different estimation results. 
4. The delay mismatching of the voltage sensor and current sensor. The ESR estimation method is based on the phase relationship and the different delay time of voltage and current sensors will result in errors. In this respect, the delay time difference should be minimized so as to achieve high accuracy.

Although there are some errors in the estimation results, the results have low dispersed degree. The aim of ESR estimation is to monitor the health of AEC, which is often considered lapsed until its ESR increases to more than two times of the initial value. Therefore, the estimation error $(<10 \%)$ is within the accepted range and the estimation results can be taken as effective indicators.

\section{Conclusions}

From a capacitor perspective, ESR calculation schemes of output capacitors for a Buck converter are studied in this paper:

(1) The degradation parameters of AECs are analyzed and ESR is indicated as the optimal health indicator.

(2) Based on the voltage-current characteristics, two ESR calculation models are proposed.

To reveal the advantages of the proposed methods, a comparison between the two proposed methods and the existing methods is presented in Table 7.

Table 7. Comparison between the Proposed Methods and the Existing Methods.

\begin{tabular}{cccc}
\hline Method & $\begin{array}{c}\text { The Existing Capacitor } \\
\text { Perspective Methods } \\
\text { (CPMs) }\end{array}$ & Proposed Method 1 & Proposed Method 2 \\
\hline Calculation model & simple & simple & simple \\
\hline Additional circuit & relatively complicated & simple & simple \\
\hline Load applicability & CCM or DCM & Both CCM and DCM & Both CCM and DCM \\
\hline Applicability & narrow & wide & wide \\
\hline
\end{tabular}

The main advantages of the two proposed methods are:

(1) Only the output voltage ripple and the inductor current ripple are sampled, which requires minimal hardware.

(2) The proposed methods apply to both CCM and DCM.

The main differences between method 1 and method 2 are:

(1) Method 1 only needs to capture two points for calculation in a switching period and method 2 needs to continuously capture several switching-period points.

(2) Method 1 is susceptive to noise and method 2 has a strong capability for noise immunity.

It should be noted that the proposed methods are only applicable to the case that the output is a single capacitor or the case that the output can be equivalent to a single capacitor. The proposed methods are not effective and need to be improved for parallel capacitors. Ultimately, we are still working on the optimal method of monitoring parallel capacitors.

Author Contributions: Writing—original draft preparation, L.R.; writing—review and editing, L.Z. and C.G.; funding acquisition, L.R. and L.Z. All authors have read and agreed to the published version of the manuscript.

Funding: This research was funded by supported by Nantong Science and Technology Plan Project, grant number JC2019095.

Conflicts of Interest: The authors declare no conflict of interest. 


\section{References}

1. Roman, E.; Alonso, R.; Ibanez, P.; Elorduizapatarietxe, S.; Goitia, D. Intelligent PV module for grid-connected PV systems. IEEE Trans. Ind. Electron. 2006, 53, 1066-1073. [CrossRef]

2. Gu, B.; Dominic, J.; Lai, J.S.; Chen, C.L.; LaBella, T.; Chen, B. High reliability and efficiency single-phase transformerless inverter for grid-connected photovoltaic systems. IEEE Trans. Power Electron. 2013, 28, 2235-2245. [CrossRef]

3. Abu-Rub, H.; Holtz, J.; Rodriguez, J.; Baoming, G. Medium-voltage multilevel converters-State of the art, challenges, and requirements in industrial applications. IEEE Trans. Ind. Electron. 2010, 57, 2581-2596. [CrossRef]

4. Gusseme, K.D.; Van de Sype, D.M.; Van den Bossche, A.P.M.; Melkebeek, J.A. Input-current distortion of CCM boost PFC converters operated in DCM. IEEE Trans. Ind. Electron. 2007, 54, 858-865. [CrossRef]

5. Yang, S.; Bryant, A.; Mawby, P.; Xiang, D.; Ran, L.; Tavner, P. An industry-based Survey of reliability in power electronic converters. IEEE Trans. Ind. Appl. 2011, 47, 1441-1451. [CrossRef]

6. Yang, S.; Xiang, D.; Bryant, A.; Mawby, P.; Ran, L.; Tavner, P. Condition monitoring for device reliability in power electronic converters: A review. IEEE Trans. Power Electron. 2010, 25, 2734-2752. [CrossRef]

7. Rastayesh, S.; Bahrebar, S.; Bahman, A.S.; Sørensen, J.D.; Blaabjerg, F. Lifetime estimation and failure risk analysis in a power stage used in wind-fuel cell hybrid energy systems. Electronics 2019, 8, 1412. [CrossRef]

8. Wang, H.; Blaabjerg, F. Reliability of capacitors for dc-link applications in power electronic converters-An overview. IEEE Trans. Ind. Appl. 2014, 50, 3569-3578. [CrossRef]

9. Algreer, M.; Armstrong, M.; Giaouris, D. Active online system identification of switch mode DC-DC power converter based on efficient recursive DCD-IIR adaptive filter. IEEE Trans. Power Electron. 2012, 27, 4425-4435. [CrossRef]

10. Li, B.X.; Low, K.S. Low sampling rate online parameters monitoring of DC-DC converters for predictive-maintenance using biogeography-based optimization. IEEE Trans. Power Electron. 2016, 31, 2870-2879. [CrossRef]

11. Miao, B.; Zane, R.; Maksimovic, D. System identification of power converters with digital control through cross-correlation methods. IEEE Trans. Power Electron. 2005, 20, 1093-1099. [CrossRef]

12. Poon, J.; Jain, P.; Spanos, C.; Panda, S.K.; Sanders, S.R. Fault prognosis for power electronics systems using adaptive parameter identification. IEEE Trans. Ind. Appl. 2017, 53, 2862-2870. [CrossRef]

13. Ma, H.; Mao, X.; Zhang, N.; Xu, D. Parameter identification of power electronics circuits based on hybrid models. In Proceedings of the 36th Power Electronics Specialists Conference, Recife, Brazil, 16 June 2005.

14. Ma, H.; Wang, L.G. Fault diagnosis and failure prediction of aluminum electrolytic capacitor in power electronic converters. In Proceedings of the 31th Annual Conference of IEEE Industrial Electronics Society, Raleigh, NC, USA, 6-10 November 2005.

15. Chen, Y.M.; Wu, H.C.; Chou, M.W.; Lee, K.Y. Online failure prediction of the electrolytic capacitor for LC filter of switching-mode power converters. IEEE Trans. Ind. Electron. 2008, 55, 400-406. [CrossRef]

16. Farjah, E.; Givi, H.; Ghanbari, T. Application of an efficient Rogowski coil sensor for switch fault diagnosis and capacitor ESR monitoring in non-isolated single-switch DC-DC converters. IEEE Trans. Power Electron. 2017, 32, 1442-1456. [CrossRef]

17. Wang, G.H.; Guan, Y.; Zhang, J.; Wu, L.; Zheng, X.; Pan, W. ESR estimation method for DC-DC converters based on improved EMD algorithm. In Proceedings of the IEEE 2012 Prognostics and System Health Management Conference, Beijing, China, 23-25 May 2012.

18. Yao, K.; Tang, W.; Hu, W.; Lyu, J. A current-sensorless online ESR and C identification method for output capacitor of buck converter. IEEE Trans. Power Electron. 2015, 30, 6993-7005. [CrossRef]

19. Yao, K.; Cao, C.; Yang, S. Noninvasive online condition monitoring of output capacitor's ESR and C for a flyback converter. IEEE Trans. Instrum. Meas. 2017, 66, 3190-3199. [CrossRef]

20. Yao, K.; Li, H.; Li, L.; Guan, C.; Li, L.; Zhang, Z.; Chen, J. A noninvasive online monitoring method of output capacitor's C and ESR for DCM flyback converter. IEEE Trans. Power Electron. 2019, 34, 5748-5763. [CrossRef]

21. Tang, S.; Dong, S.; Liu, Y.; Zhang, Q. Current-sensorless online ESR monitoring of capacitors in boost converter. J. Eng. 2019, 16, 2569-2754. [CrossRef]

22. Ahmad, M.W.; Agarwal, N.; Anand, S. Online monitoring technique for aluminum electrolytic capacitor in solar PV-based DC system. IEEE Trans. Ind. Electron. 2016, 63, 7059-7066. [CrossRef] 
23. Vogelsberger, M.A.; Wiesinger, T.; Ertl, H. Life-cycle monitoring and voltage-managing unit for DC-link electrolytic capacitors in PWM converters. IEEE Trans. Power Electron. 2011, 26, 493-503. [CrossRef]

24. Pang, H.M.; Bryan, P.M.H. A life prediction scheme for electrolytic capacitors in power converters without current sensor. In Proceedings of the 25th Annual IEEE Applied Power Electronics Conference and Exposition, Palm Springs, CA, USA, 21-25 February 2010.

25. Hannonen, J.; Honkanen, J.; Ström, J.P.; Kärkkäinen, T.; Räisänen, S.; Silventoinen, P. Capacitor aging detection in a DC-DC converter output stage. IEEE Trans. Ind. Appl. 2016, 52, 3224-3233. [CrossRef]

26. Sun, P.; Gong, C.; Du, X.; Luo, Q.; Wang, H.; Zhou, L. Online condition monitoring for both IGBT module and DC-link capacitor of power converter based on short-circuit current simultaneously. IEEE Trans. Ind. Electron. 2017, 64, 3662-3671. [CrossRef]

(C) 2020 by the authors. Licensee MDPI, Basel, Switzerland. This article is an open access article distributed under the terms and conditions of the Creative Commons Attribution (CC BY) license (http://creativecommons.org/licenses/by/4.0/). 